\title{
PRELIMINARY RESULTS FROM OBSERVATIONS WITH THE FOURIER TACHOMETER
}

\author{
J. SCHOU \\ Boulder, Colorado, USA \\ T.M. BROWN \\ High Altitude Observatory \\ K.T. BACHMANN \\ High Altitude Observatory
}

Institut for Fysik og Astronomi, Arhus Universitet, Denmark, and High Altitude Observatory, National Center for Atmospheric Research ",

\section{INTRODUCTION}

Recently several interesting results concerning the time dependence of solar p-mode frequencies have appeared. In particular it has been shown that the mode frequencies change with solar activity (Libbrecht and Woodard 1991).

We present preliminary results from an analysis of two $\approx 3$ month observation runs with the Fourier Tachometer (which was operated by HAO and NSO) from 1987 and 1989. The analysis was done with two different methods in order to test for systematic errors. We show that the mode frequencies change in a manner similar to that reported by Libbrecht and Woodard. We also present results for the frequency splittings caused by the solar rotation.

\section{RESULTS}

In both analysis methods the apodized velocity images are first projected onto a set of masks to isolate individual $(m, l)$ 's. These time-series are then Fourier transformed. In the analysis method used for the results presented here a simultaneous maximum likelihood fit is done to all $m$ 's in a frequency range around each target $(l, n)$. The fit is at each $m$ a background plus a Lorenzian with a linewidth independent of $m$, a mode amplitude and a frequency of

$$
\nu_{n l m}=\nu_{n l}+L \sum_{i=1}^{6} a_{i} P_{i}^{\prime}\left(\frac{m}{L}\right)
$$

- The National Center for Atmospheric Research is sponsored by the National Science Foundation. 
where the $P$ 's are polynomials similar to Legendre polynomials but designed to be orthogonal with respect to summation. The leakage from modes with different $n, l$ or $m$ is taken into account together with the correlations between $m$ 's introduced by this leakage. The other method does a simpler least squares fit not taking all the leakages etc. into account. The two methods generally give similar results. The differences, although systematic, are not large enough to be seen on most plots, and the interpretations given here do not depend on the method used.

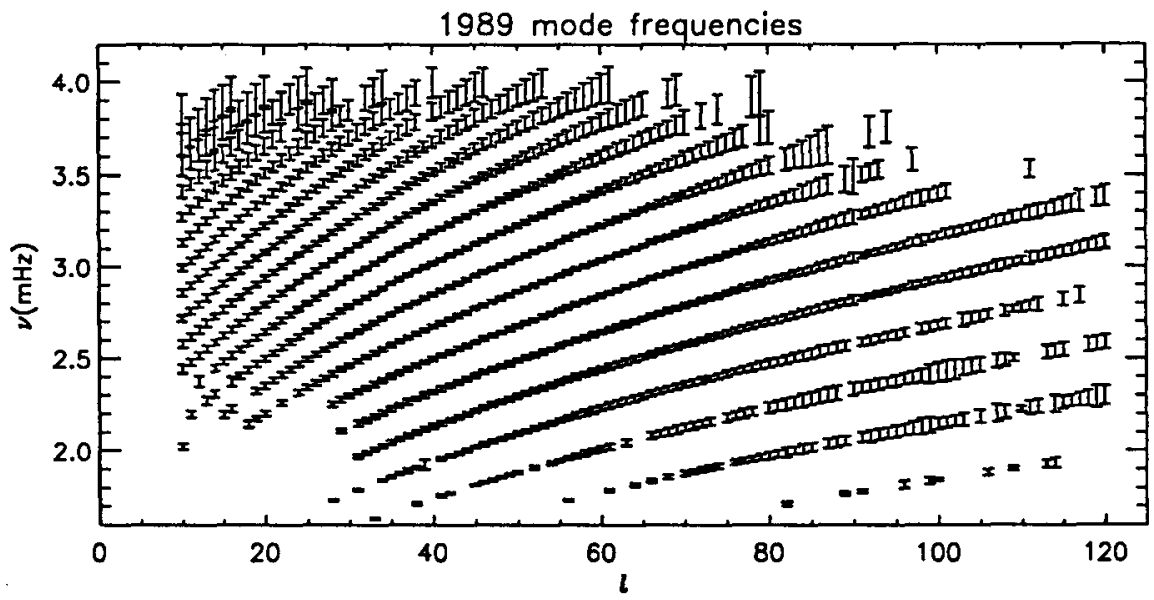

Fig. 1. The modes successfully fit for 1989. The error bars are 250 times the estimated standard errors.

\section{DISCUSSION}

The frequency differences in figure 2 are qualitatively similar to those of Libbrecht and Woodard, confirming their results. In particular it appears that most of the frequency changes scale like the inverse mode mass and hence are caused by perturbations in the structure close to the solar surface.

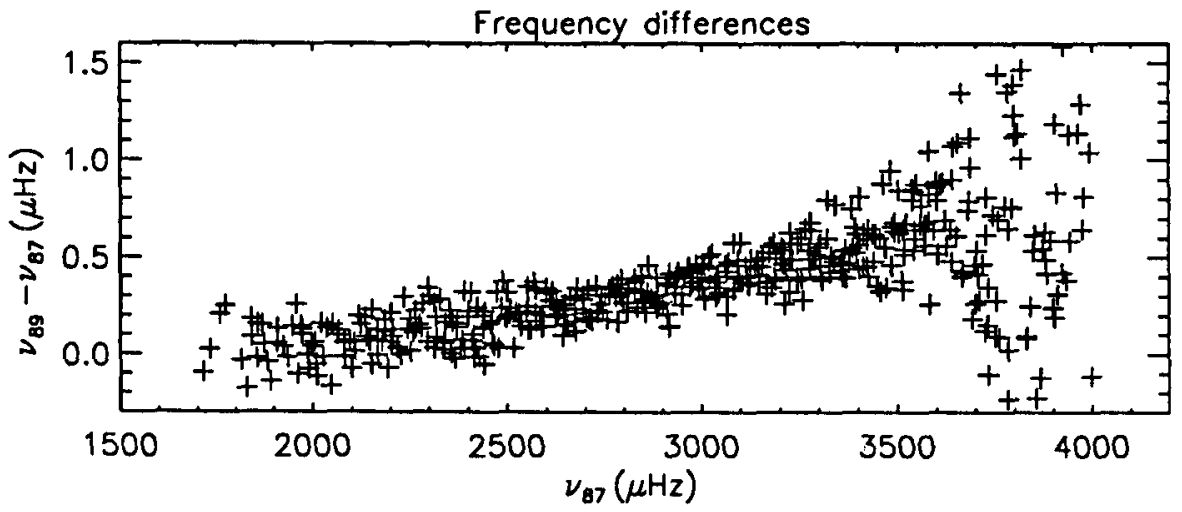

Fig. 2. The difference in the mode frequencies between the two years. Only modes with $20 \leq l \leq 60$ have been shown to decrease the scatter. 


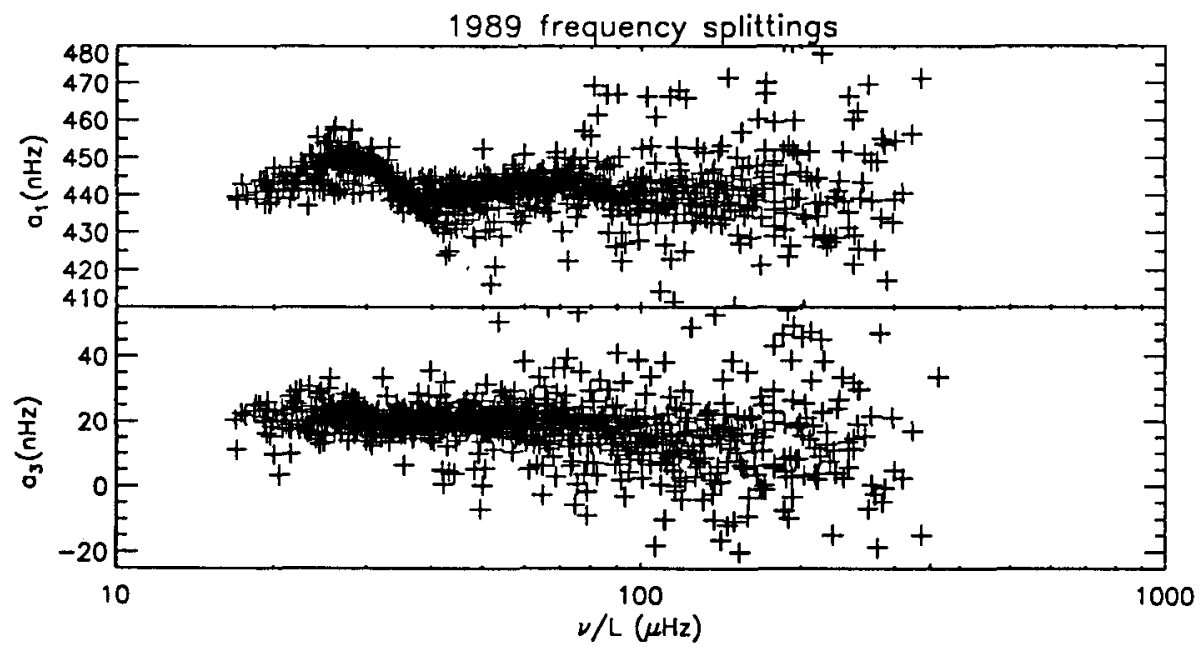

Fig. 3. $a_{1}$ and $a_{3}$ from the 1989 dataset as a function of $\nu / L$.

The plot of $a_{1}$ as a function of $\nu / L$ (which is a measure of the turning point depth, and thus the region the mode samples) contain some interesting features below $\nu / L<40 \mu \mathrm{Hz}$. We have not yet been able to identify the cause of these features. In particular we have no evidence that they are artifacts of the analysis methods used. A reason to be concerned, however, is that the region of the bump $25 \mu \mathrm{Hz} \leq \nu / L \leq 40 \mu \mathrm{Hz}$ corresponds with the range in which the frequency spacing between two neighbouring l's is equal to (1day) $)^{-1}=$ $11.57 \mu \mathrm{Hz}$ within a mode linewidth. Since the daily observation cycle causes sidelobes to appear around a given mode with this spacing and the individual $(l, m)$ 's are not perfectly isolated, this could potentially cause problems. For the 1989 data we have tried such tests as dividing the time-series in three parts and analyzing them individually, decreasing the duty cycle of the observations (thereby increasing the 1 /day sidelobes), changing the initial estimates for the mode parameters, change the assumed radius of the Sun, and distorting the images in various ways. None of this makes any significant changes. It is also interesting that the feature is very different in 1987.

The standard interpretation of the $a$ 's is that they are caused by the solar rotation. If they are inverted to obtain the internal solar rotation it turns out that it shows large oscillations as a function af radius. If this result for the rotation holds, it would indeed be very interesting. It is, however, such an extreme result that we feel reluctant to accept it. A comparison of results from several years and different instruments would seem useful before drawing wideranging conclusions from this result.

\section{REFERENCES}

Libbrecht, K. G. and Woodard, M. F., 1991, Science, 253, 152. 